University of New Orleans

ScholarWorks@UNO

4-1979

\title{
Transformation of Fresnel's interface reflection and transmission coefficients between normal and oblique incidence
}

R. M.A. Azzam

University of New Orleans, razzam@uno.edu

Follow this and additional works at: https://scholarworks.uno.edu/ee_facpubs

Part of the Electrical and Electronics Commons, and the Physics Commons

\section{Recommended Citation}

R. M. A. Azzam, "Transformation of Fresnel's interface reflection and transmission coefficients between normal and oblique incidence," J. Opt. Soc. Am. 69, 590-596 (1979)

This Article is brought to you for free and open access by the Department of Electrical Engineering at ScholarWorks@UNO. It has been accepted for inclusion in Electrical Engineering Faculty Publications by an authorized administrator of ScholarWorks@UNO. For more information, please contact scholarworks@uno.edu. 


\title{
Transformation of Fresnel's interface reflection and transmission coefficients between normal and oblique incidence
}

\author{
R. M. A. Azzam \\ Hematology Division and Department of Internal Medicine, The University of Nebraska Medical Center, Omaha, Nebraska 68105 \\ (Received 9 December 1977)

\begin{abstract}
If $w$ denotes an interface Fresnel reflection or transmission coefficient for $s$ - or $p$-polarized light at an oblique angle of incidence $\phi$, and $z$ denotes the same coefficient at normal incidence, we find that $w$ is an analytic function of $z, w=f(z)$, that depends on $\phi$ but not on the specific optical properties of the two media on both sides of the interface. All four functions that correspond to the four distinct Fresnel coefficients and their inverses are determined. We single out for detailed examination, as an example, the relationship between the reflection of $s$-polarized light at $45^{\circ}$ angle of incidence and at normal incidence for any transparent medium/absorbing medium interface by considering the mapping properties of the associated transformation and its inverse between the $z$ and $w$ planes. A useful byproduct of this investigation is a technique for the determination of the optical properties of isotropic and uniaxially and biaxially anisotropic absorbing media from measurement of reflectance at normal and oblique incidence.
\end{abstract}

\section{INTRODUCTION}

The reflection and transmission of a plane wave of light (or any other electromagnetic radiation) at the planar interface between two homogeneous and isotropic media are governed by the well-known Fresnel coefficients. ${ }^{1}$ These fundamental coefficients also play a key role in the theory of light reflection and transmission by stratified multilayer media.

In this paper we show that if $z$ and $w$ represent any Fresnel coefficient at normal and oblique incidence, respectively, then $w$ is an analytic function of $z, w=f(z)$, that depends parametrically on the angle of incidence $\phi$. In such a function the specific optical properties (complex refractive indices) of the two media that surround the interface do not appear. We determine the function $w=f(z)$ and its inverse $z=g(w)$ for the reflection and transmission of light linearly polarized parallel $(p)$ and perpendicular $(s)$ to the plane of incidence. We illustrate the mapping between the complex $z$ and $w$ planes by one of the Fresnel coefficients (for $s$ reflection) at normal and one oblique angle of incidence $\left(45^{\circ}\right)$. As an application, we show how the optical properties of isotropic and uniaxially and biaxially anisotropic absorbing media can be determined from measurements of reflectance at normal and oblique incidence.

\section{TRANSFORMATION OF THE FRESNEL COEFFICIENTS FROM NORMAL TO OBLIQUE INCIDENCE}

Let light be incident at an angle $\phi$ from a medium of refractive index $n$ onto its planar interface with another medium with refractive index $N$, Fig. 1 . If we define

$$
\nu=N / n
$$

to be the ratio of the refractive indices of the two media, we find that the complex-amplitude Fresnel reflection $(r)$ and transmission $(t)$ coefficients $^{2}$ are functions of $\nu$ only given by

$$
\begin{gathered}
r_{\phi}^{s}=\frac{\cos \phi-\left(\nu^{2}-\sin ^{2} \phi\right)^{1 / 2}}{\cos \phi+\left(\nu^{2}-\sin ^{2} \phi\right)^{1 / 2}}, \\
r_{\phi}^{p}=\frac{\nu^{2} \cos \phi-\left(\nu^{2}-\sin ^{2} \phi\right)^{1 / 2}}{\nu^{2} \cos \phi+\left(\nu^{2}-\sin ^{2} \phi\right)^{1 / 2}}, \\
t_{\phi}^{s}=\frac{2 \cos \phi}{\cos \phi+\left(\nu^{2}-\sin ^{2} \phi\right)^{1 / 2}}, \\
t_{\phi}^{p}=\frac{2 \nu \cos \phi}{\nu^{2} \cos \phi+\left(\nu^{2}-\sin ^{2} \phi\right)^{1 / 2}} .
\end{gathered}
$$

Equations (2) and (3) are valid when the two media are absorbing in which case $n, N$, and $\phi$ are all complex. However, we are often interested in the special case when the medium of incidence is transparent so that $n$ and $\phi$ are real. That the Fresnel coefficients at an oblique angle of incidence $\phi$ are determined only by the refractive index ratio $\nu$ is an important point that is not usually recognized. ${ }^{3}$

The Fresnel coefficients at normal incidence are obtained by setting $\phi=0$ in Eqs. (2) and (3); this gives

$$
\begin{gathered}
r_{0}^{s}=-r_{0}^{p}=(1-\nu) /(1+\nu), \\
t_{0}^{s}=t_{0}^{p}=2 /(1+\nu) .
\end{gathered}
$$

The $p$ and $s$ linear polarizations are physically indistinguishable at normal incidence and the difference in sign of their reflection coefficients, Eq. (4), arises because in Fig. 1 the $p$ directions for the incident and reflected waves become antiparallel, while the $s$ directions are parallel, at normal incidence. $^{4}$

If we denote a particular Fresnel coefficient at oblique incidence by $w$ and the same coefficient ${ }^{5}$ at normal incidence by $z$, we see from Eqs. (2)-(5) that both $w$ and $z$ are functions of $\nu$. Elimination of $\nu$ between $w$ and $z$ provides the direct relationship (transformation), $w=f(z)$. The desired transformations thus obtained are all summarized in the following.

For the reflection of $s$-polarized light, the Fresnel coefficient 


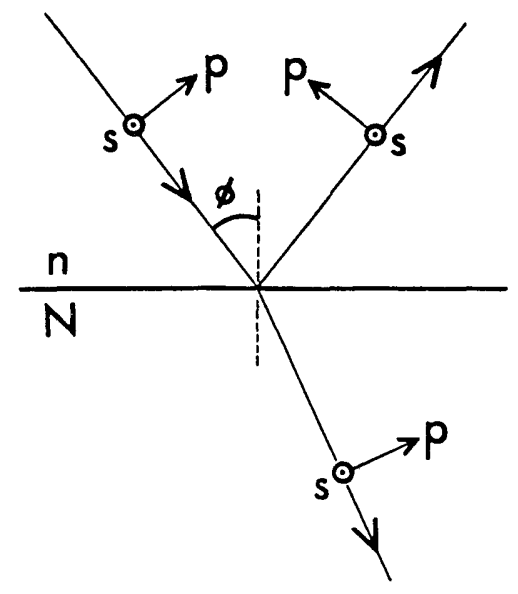

FIG. 1. The reflection and transmission (refraction) of a plane wave of light at the planar interface between two media with refractive indices $n$ and $N . \quad \phi$ is the angle of incidence and $p$ and $s$ are the two principal polarization directions parallel and perpendicular to the plane of incidence, respectively.

at oblique incidence $w$ is related to the same coefficient at normal incidence $z$ by

$$
w=\frac{(1+z)-\left(z^{2}-a z+1\right)^{1 / 2}}{(1+z)+\left(z^{2}-a z+1\right)^{1 / 2}}
$$

and the corresponding transformation for the reflection of $p$-polarized light is

$$
w=\frac{(1+z)^{2}-(1-z)\left(1+a z+z^{2}\right)^{1 / 2}}{(1+z)^{2}+(1-z)\left(1+a z+z^{2}\right)^{1 / 2}},
$$

where, in Eqs. (6) and (7),

$$
a=2\left(2 \tan ^{2} \phi+1\right) .
$$

For the transmission of $s$-polarized light, the transformation of the Fresnel coefficient from normal to oblique incidence is

$$
w=2 z /\left[z+\left(z^{2}-4 c z+4 c\right)^{1 / 2}\right],
$$

and the corresponding transformation for the transmission of $p$-polarized light is

$$
w=\frac{2 z(2-z)}{(2-z)^{2}+z\left(z^{2}-4 c z+4 c\right)^{1 / 2}},
$$

where, in Eqs. (9) and (10),

$$
c=\sec ^{2} \phi .
$$

\section{INVERSE TRANSFORMATIONS}

It is also of interest to derive the inverse transformations that determine the Fresnel coefficients at normal incidence $(z)$ from their corresponding values at oblique incidence $(w)$. Algebraic manipulations (omitted here) lead to the following results.

For $s$ reflection, the inverse of Eq. (6) is

$$
z=\frac{(a+2 p) \pm\left[\left(a^{2}-4\right)+4(a+2) p\right]^{1 / 2}}{2(1-p)},
$$

where

$$
p=[(1-w) /(1+w)]^{2},
$$

and $a$ is given by Eq. (8). For $p$ reflection, the inverse of Eq. (7) is obtained by solving the following quartic equation for $z$ :

$$
\alpha_{4} z^{4}+\alpha_{3} z^{3}+\alpha_{2} z^{2}+\alpha_{1} z+\alpha_{0}=0,
$$

where

$$
\begin{gathered}
\alpha_{0}=\alpha_{4}=p-1, \\
\alpha_{1}=\alpha_{3}=4 p-a+2, \\
\alpha_{2}=6 p+2 a-2,
\end{gathered}
$$

and $a$ and $p$ are given by Eqs. (8) and (13), respectively.

For $s$ transmission, inversion of Eq. (9) yields

$$
z=\frac{-2 c \pm 2\left[c^{2}+c\left(q^{2}-1\right)\right]^{1 / 2}}{q^{2}-1}
$$

where

$$
q=(2-w) / w
$$

and $c$ is given by Eq. (11). Finally, for $p$ transmission, inversion of Eq. (10) gives the following quartic in $z$ :

$$
\beta_{4} z^{4}+\beta_{3} z^{3}+\beta_{2} z^{2}+\beta_{1} z+\beta_{0}=0,
$$

where

$$
\begin{gathered}
\beta_{0}=4 w^{2}, \\
\beta_{1}=-8 w^{2}-8 w, \\
\beta_{2}=(6-c) w^{2}+12 w+4, \\
\beta_{3}=(c-2) w^{2}-6 w-4, \\
\beta_{4}=w+1,
\end{gathered}
$$

and $c$ is given by Eq. (11).
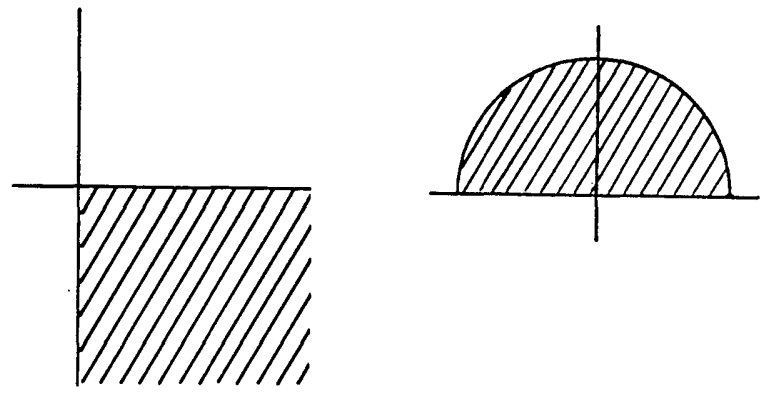

$\nu$ plane
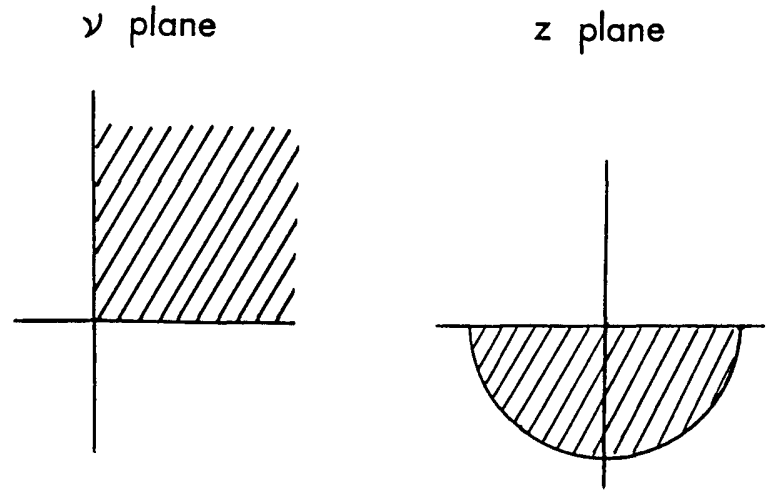

FIG. 2. Mapping between the complex plane of the refractive index ratio $\nu$ and the complex plane of the normal-incidence s-reflection coefficient $z$. 


\section{MAPPING OF THE FRESNEL REFLECTION COEFFICIENT FOR $s$-POLARIZED LIGHT BETWEEN NORMAL AND OBLIQUE INCIDENCE: AN EXAMPLE}

Much insight into the nature of a complex transformation governed by an analytic function connecting two complex variables $z$ and $w$ can be gained if we consider the mapping (imaging) of geometrical features in the $z$ plane onto the $w$ plane and vice versa.

As we have seen in Secs. II and III, the four distinct Fresnel coefficients lead to eight analytic functions (transformations) given by Eqs. (6), (7), (9), (10), (12), (14), (16), and (18). Examination of the mapping by these eight functions of the family of concentric circles with the origin as center $(|z|$ or $|w|$ $=$ const) and the orthogonal family of straight lines through the origin ( $\arg z$ or $\arg w=$ const) leads to a large and fascinating collection of orthogonal sets of curves. Here we discuss only as an example the mapping of the Fresnel reflection coefficient for the $s$ polarization between normal and $45^{\circ}$ oblique incidence [Eqs. (6) and (12) with $\phi=45^{\circ}$ ].

We assume that the medium of incidence is transparent so that its refractive index $n$ is real and that the second medium is in general absorbing so that $N$ and $\nu$ are complex.

If we choose the $e^{j \omega t}$ time dependence for the harmonic (monochromatic) incident wave, the complex refractive index ratio $\nu$ [Eq . (1)] is then limited to the fourth quadrant of the $\nu$ plane. The bilinear transformation, $z=(1-\nu) /(1+\nu)$, that links the normal-incidence $s$-reflection coefficient $z$ to $\nu$, maps the fourth quadrant of the $\nu$ plane into the upper half of the unit circle in the $z$ plane. If $e^{-j \omega t}$ is chosen instead, $\nu$ will occupy the first quadrant of the $\nu$ plane which is mapped onto the lower half of the unit circle in the $z$ plane (when $\nu=\nu^{*}, z$ $\left.=z^{*}\right)$, Fig. 2. Although it is sufficient, when considering the mapping properties of Eq. (6), to limit $z$ inside and on the boundaries of either the upper or lower half of the unit circle in the $z$ plane, we will allow $z$ to assume values inside and on the full unit circle. This means that both forms of the time dependence $e^{j \omega t}$ and $e^{-j \omega t}$ are simultaneously represented. ${ }^{6}$

The following properties of the transformation given by Eq. (6), that relates the reflection of $s$-polarized light at normal and oblique incidence, can be readily verified.

(1) The transformation is double valued. For a given value of $z$, there are two corresponding values $w_{1}$ and $w_{2}$ of $w$ [because of the two values of the square root in Eq. (6)] such that $w_{2}=1 / w_{1}$. Only that value of $w$ for which $|w| \leqslant 1$ (i.e., that lies inside or on the unit circle $|w|=1$ in the $w$ plane) is selected as physically acceptable. The other value, for which $|w|>1$, is discarded.

(2) When $z=z^{*}$, we find that $w=w^{*}$. Therefore, mapping between the upper halves of the complex $z$ and $w$ planes determines mapping of the lower halves by mirror reflection in the real axis.

(3) When $z=0$ and $z=-1$, we have $w=0$ and $w=-1$, respectively. ${ }^{7}$ It can be proved that these two points are the only invariant points of the transformation.

(4) There is one-to-one correspondence between points inside and on the unit circle $|z|=1$ in the $z$ plane with points
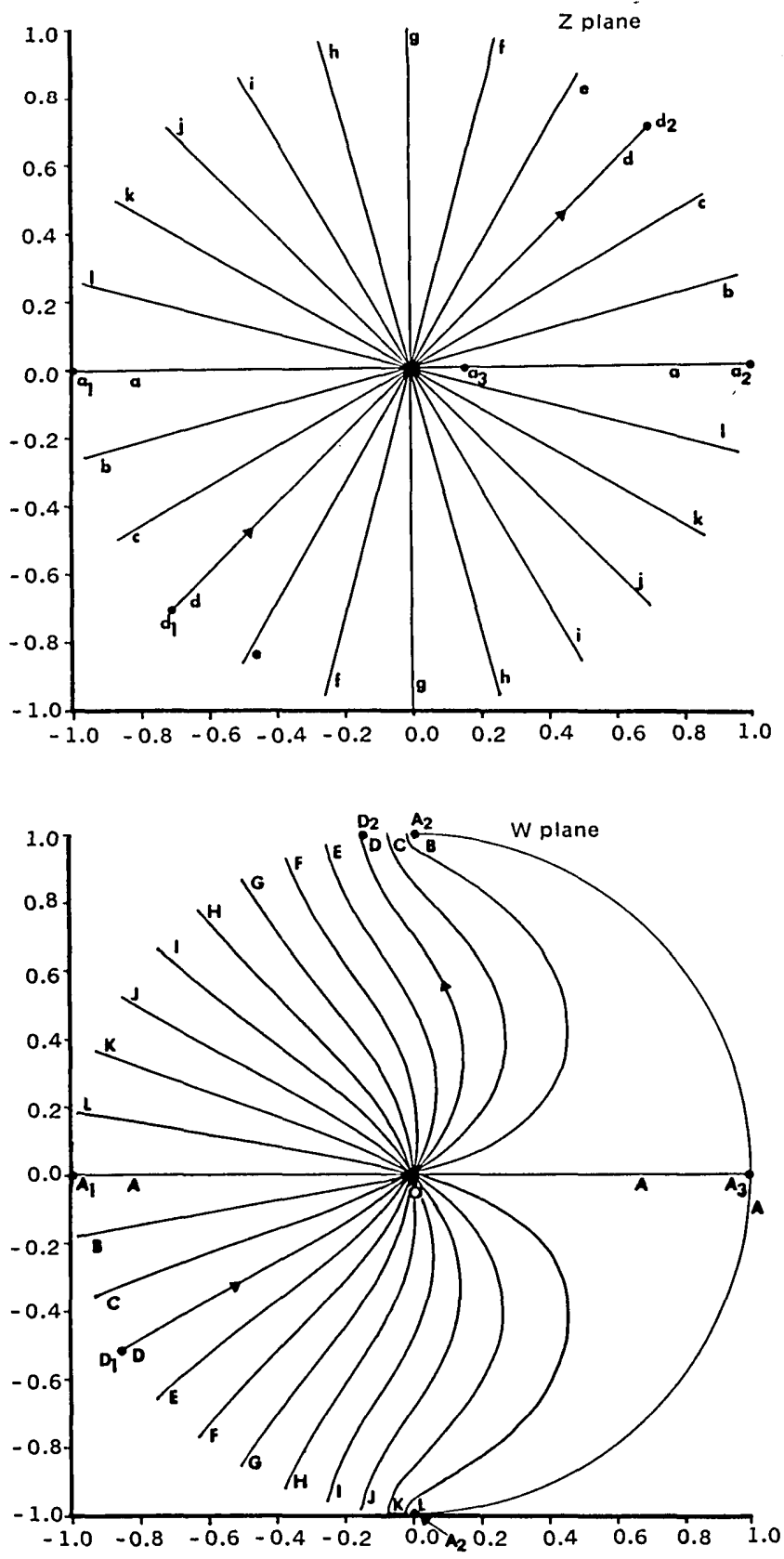

FIG. 3. Mapping of the Fresnel reflection coefficient for s-polarized light between normal incidence ( $z$ plane) and $45^{\circ}$ oblique incidence ( $w$ plane). The straight lines through the origin $a, b, c, \ldots$ lin the $z$ plane correspond to $\arg z=\left(0,180^{\circ}\right),\left(15^{\circ}, 195^{\circ}\right),\left(30^{\circ}, 210^{\circ}\right), \ldots,\left(165^{\circ}, 345^{\circ}\right)$, respectively, and their images in the $w$ plane are marked by the corresponding capital letters $A, B, C, \ldots, L$. As $z$ scans line $d$ from $d_{1}$ to $d_{2}, w$ scans its image $D$ from $D_{1}$ to $D_{2}$.

inside and on the unit circle $|w|=1$ in the $w$ plane. If $z$ scans the segment of the real axis between $z=-1$ and $z=(1-$ $\sin \phi) /(1+\sin \phi)$ in the $z$ plane, $w$ will scan the segment of the real axis between $w=-1$ and $w=+1$ in the $w$ plane. The remaining segment of the real axis of the $z$ plane inside the unit circle, between $z=(1-\sin \phi) /(1+\sin \phi)$ and $z=+1$, is mapped either onto the arc of the unit circle of the $w$ plane between $w=+1$ and $w=e^{j 2 \phi}$ (when the $e^{j \omega t}$ time dependence is assumed) or the image arc between $w=+1$ and $w=e^{-j 2 \phi}$ 

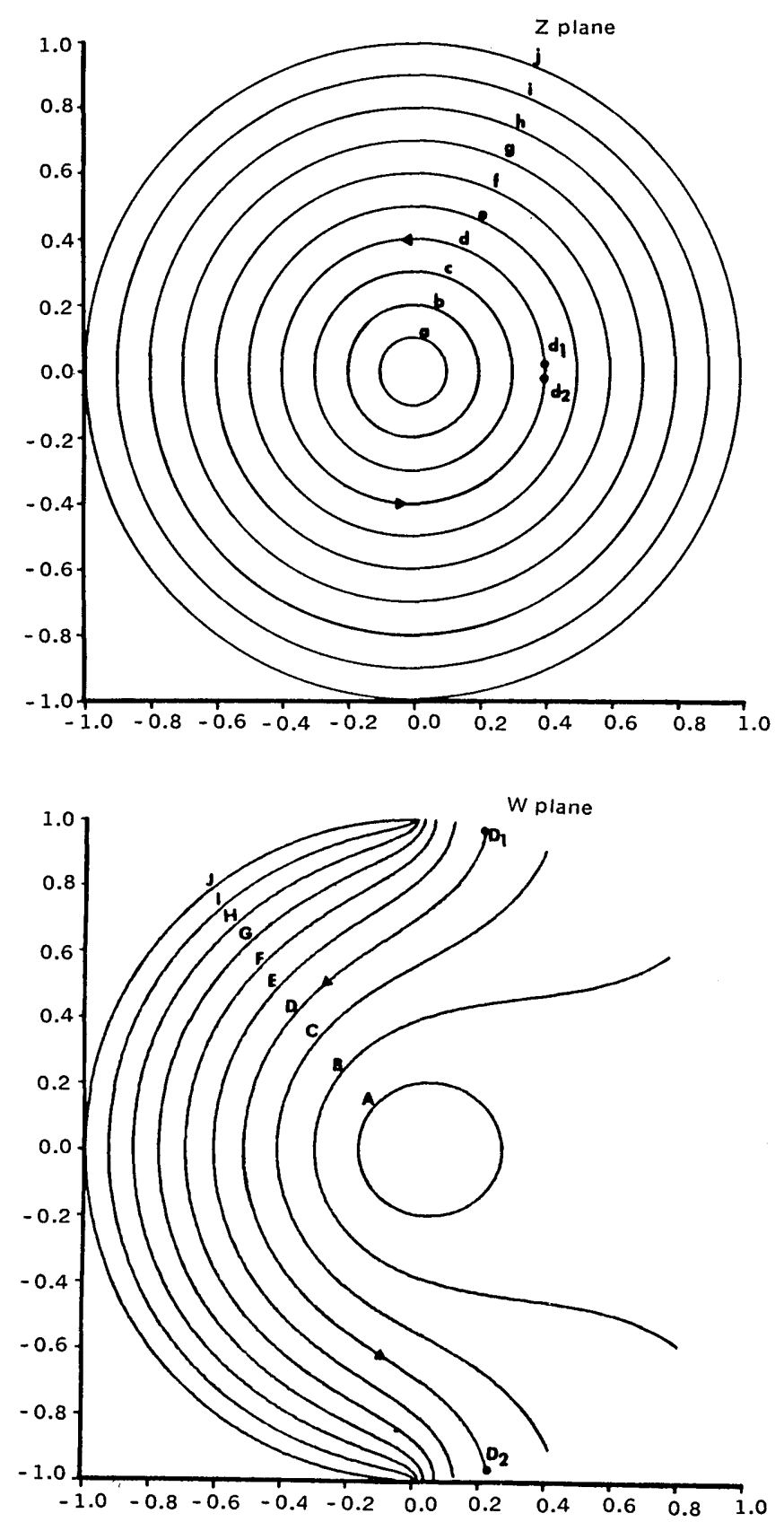

FIG. 4. Mapping of the Fresnel reflection coefficient for s-polarized light between normal incidence ( $z$ plane) and $45^{\circ}$ oblique incidence. ( $w$ plane). The circles centered on the origin $a, b, c, \ldots, j$ in the $z$ plane correspond to $|z|=0.1,0.2,0.3, \ldots, 1$ and their images in the $w$ plane are marked by $A, B, C, \ldots, J$, respectively. As the circle $d$ in the $z$ plane is traced from $d_{1}$ to $d_{2}$, its image $D$ in the $w$ plane is traced from $D_{1}$ to $D_{2}$, in the directions of the indicated arrows. All circles $0<|z| \leqslant 0.1716$ in the $z$ plane are imaged into closed contours in the $w$ plane $[0.1716=(1-\sin \phi) /(1+\sin \phi)$ where $\phi=45^{\circ}$ is the angle of incidence].

(when $e^{-j \omega t}$ is chosen instead). ${ }^{8}$ If $z$ traces the upper half of the circumference of the unit circle (i.e., $|z|=1$ and $0 \leqslant \arg z$ $\leqslant \pi$ ) from $z=+1$ to $z=-1, w$ will trace the arc of the unit circle in the $w$ plane between $w=e^{j 2 \phi}$ and $w=-1$ (i.e., the arc $|w|=1$ and $2 \phi \leqslant \arg w \leqslant \pi$ ). The lower half of the circumference of the unit circle in the $z$ plane between $z=+1$ and $z=-1$ is mapped onto the arc of the unit circle in the $w$ plane
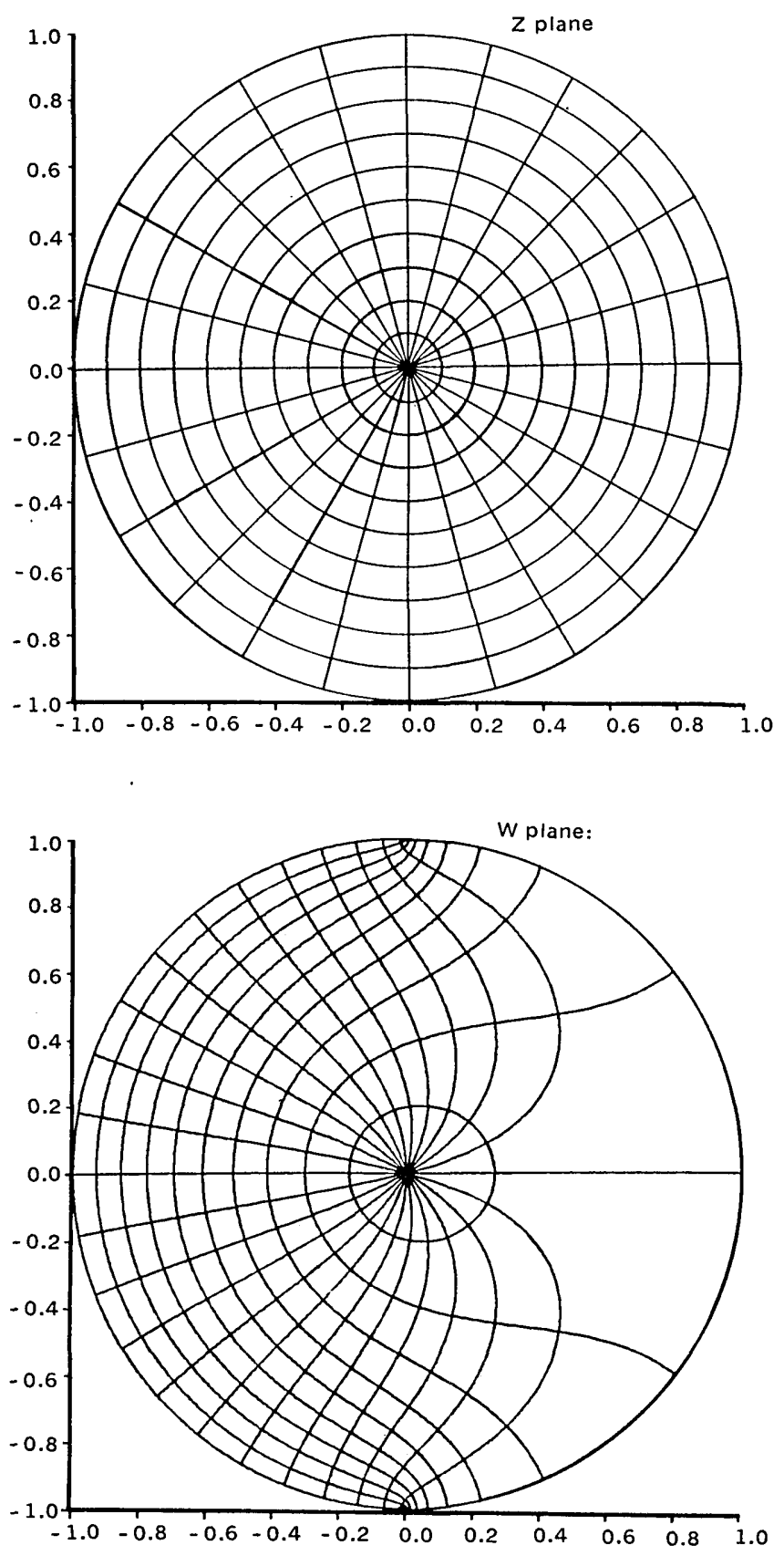

FIG. 5. The superposition of Figs. 3 and 4 produces orthogonal sets of contours in the $z$ and $w$ planes.

between $w=e^{-j 2 \phi}$ and $w=-1 . .^{9} \quad$ Furthermore, the interior of the upper and lower halves of the unit circle in the $z$ plane are mapped onto the interior of the upper and lower halves of the unit circle in the $w$ plane, respectively.

The detailed $z \rightarrow w$ mapping properties of Eq. (6) are illustrated by Figs. $3-5$. Figure 3 shows a family of angularly equispaced $\left(15^{\circ}\right.$ apart) straight lines through the origin of the $z$ plane $^{10}$ (left) and their images in the $w$ plane (right). Points and curves that are images of one another are marked by the same (small and capital) letters. Figure 4 shows a family of concentric circles of radii $0.1,0.2,0.3, \ldots, 1$ centered on the origin in the $z$ plane $^{11}$ (left) and their images in the $w$ plane 

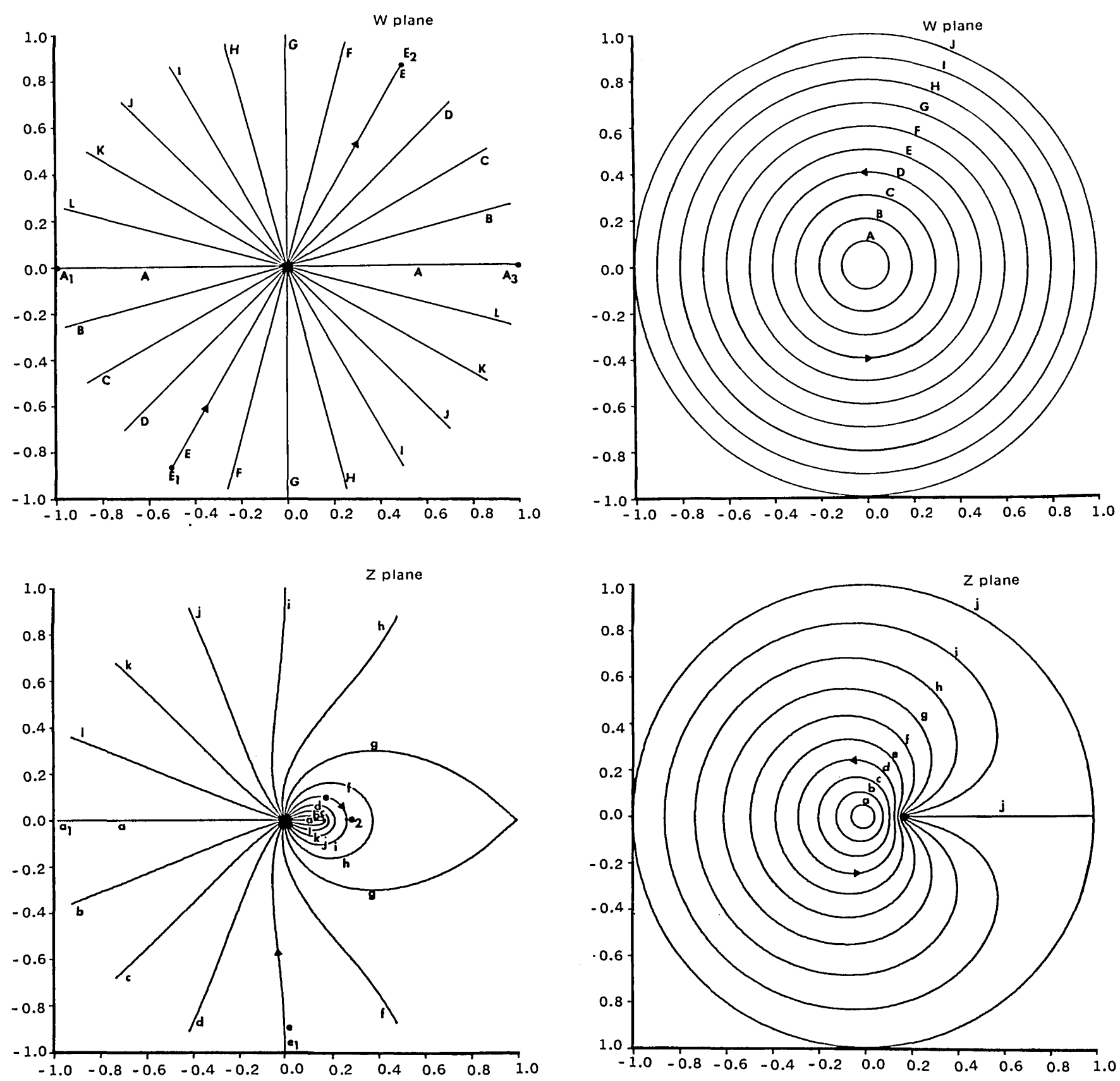

FIG. 6. Inverse mapping of the Fresnel reflection coefficient for s-polarized light between oblique incidence at $45^{\circ}$ ( $w$ plane) and normal incidence ( $z$ plane). The straight lines through the origin of the $w$ plane $A, B, C, \ldots$, $L$ correspond to $\arg w=\left(0,180^{\circ}\right),\left(15^{\circ}, 195^{\circ}\right),\left(30^{\circ}, 210^{\circ}\right), \ldots,\left(165^{\circ}\right.$, $345^{\circ}$ ) and their images in the $z$ plane are denoted by $a, b, c, \ldots, l$, respectively.

(right). Figure 5 is a superposition of Figs. 3 and 4 and shows that the orthogonal (polar) set of straight lines and circles through and around the origin in the $z$ plane, is mapped onto orthogonal sets of curves in the $w$ plane, as is expected from a conformal mapping (by and analytic function).

The mapping properties of the inverse transformation $w$ $\rightarrow z$, which is given by Eq. (12), are illustrated by Figs. $6-8$ which are self-explanatory.

FIG. 7. Inverse mapping of the Fresnel reflection coefficient for s-polarized light between oblique incidence at $45^{\circ}$ ( $w$ plane) and normal incidence ( $z$ plane). The circles $A, B, C, \ldots, J$ in the $w$ plane correspond to $|w|=0.1$, $0.2,0.3, \ldots, 1$ and their images in the $z$ plane are $a, b, c, \ldots, j$, respectively.

\section{A TWO-REFLECTANCE METHOD (TRM) FOR THE DETERMINATION OF THE OPTICAL PROPERTIES OF ISOTROPIC AND ANISOTROPIC MEDIA}

The study of the transformation of the Fresnel coefficients between normal and oblique incidence is significant not only because it provides new insight into the nature and behavior of these fundamental coefficients, but also because such insight can lead to new conclusions. In this section we provide an example. 

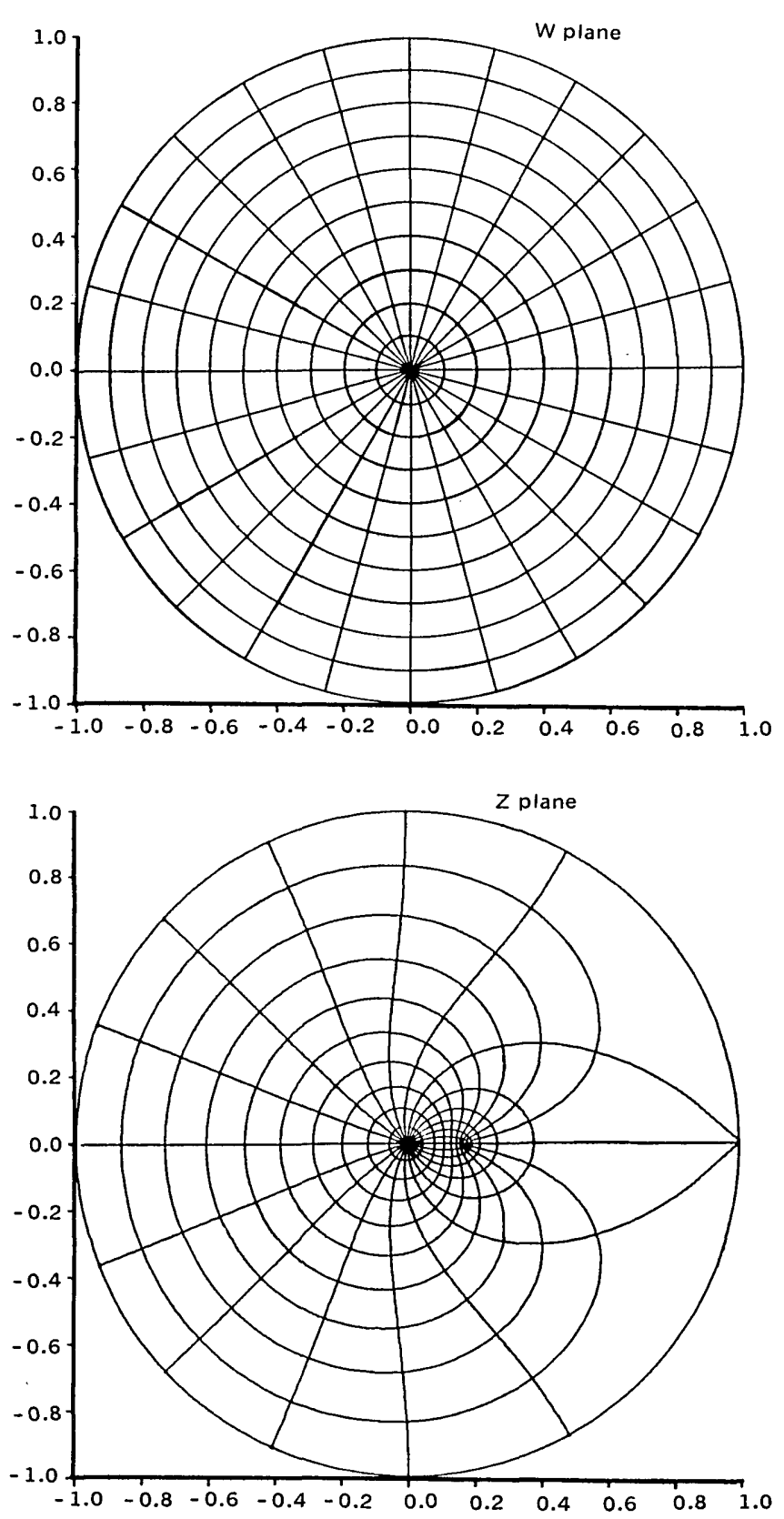

FIG. 8. The superposition of Figs. 6 and 7 yields orthoganal sets of curves in the $w$ and $z$ planes.

Assume that linearly $s$-polarized light is incident from an isotropic medium of known refractive index (e.g., air or vacuum) onto the planar surface of an isotropic absorbing medium. If we measure the surface (intensity) reflectances $\mathscr{R}_{0}$ and $\mathcal{R}_{\phi}$ at normal and one oblique angle of incidence $\phi$, we readily determine $|z|=\mathcal{R}_{0}^{1 / 2}$ and $|w|=\mathcal{R}_{\phi}^{1 / 2}$. If we draw in the complex $z$ plane the circle $|z|=\mathcal{R}_{0}^{1 / 2}$ and the image of $|w|$ $=\mathscr{R}_{\phi}^{1 / 2}$, the points of intersection of these two curves determine the complex normal-incidence reflection coefficient $z$. [Thus measurement of reflectance at oblique incidence leads, through the foregoing construction, to the specification of the phase shift (argz) upon normal-incidence reflection.]

Figure 9 illustrates how this technique works for a case when the measured reflectances for $s$-polarized light are assumed

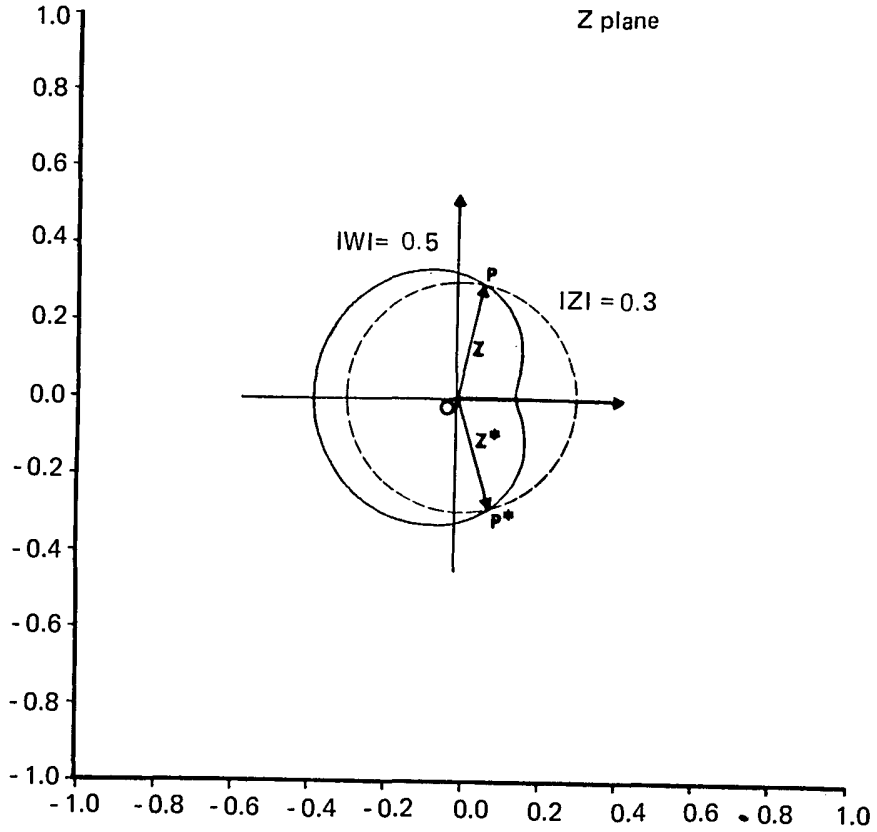

FIG. 9. Geometrical construction to show how the complex Fresnel reflection coefficient for s-polarized light at normal incidence $\left(z\right.$ or $\left.z^{*}\right)$ can be determined from two intensity reflectances measured at normal and $45^{\circ}$ oblique incidence (shown here to be $|z|^{2}=0.09$ and $|w|^{2}=0.25$, respectively).

to be $\mathcal{R}_{0}=0.09$ and $\mathcal{R}_{\phi}\left(\phi=45^{\circ}\right)=0.25$. Notice that the $|z|$ $=0.3$ circle intersects the image of $|w|=0.5$ (at $\phi=45^{\circ}$ ) at two points $P$ and $P^{*}$ with associated complex-conjugate numbers $z$ and $z^{*}$. The complex refractive index ratio $\nu$ can subsequently be easily computed from $\nu=(1-z) /(1+z)$, or determined graphically using the so-called Malé nomogram. ${ }^{12}$

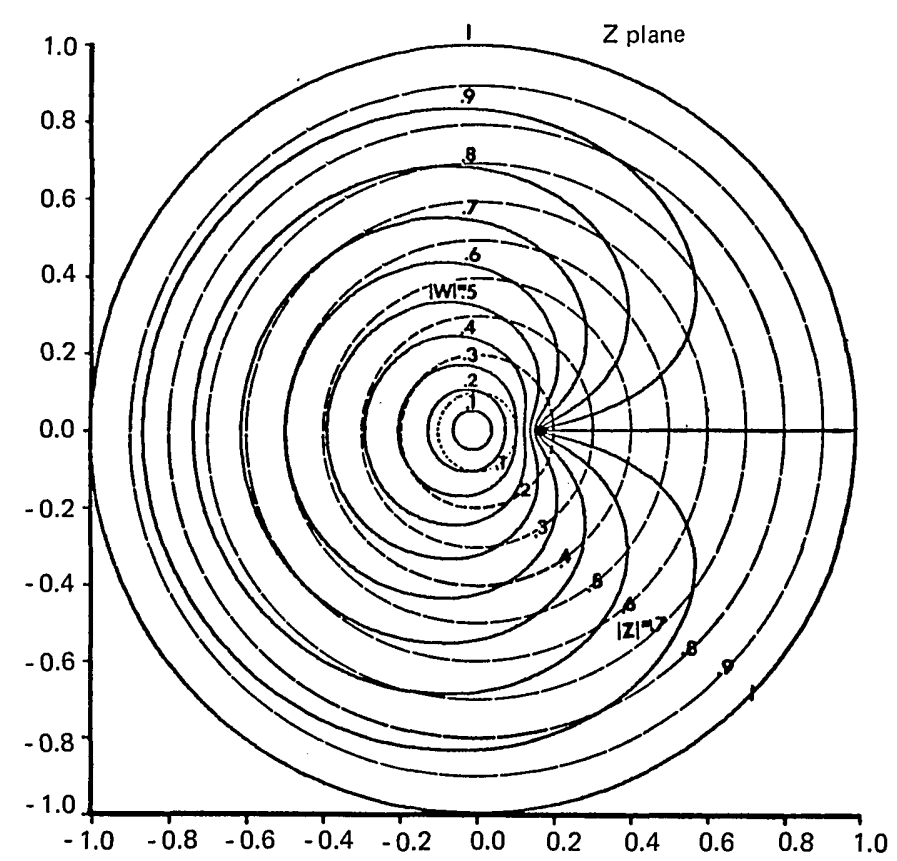

FIG. 10. Nomogram for applying the two-reflectance method (TRM), using $s$-polarized light at normal and $45^{\circ}$ incidence, to determine the complex normal-incidence reflection coefficient (hence the optical properties of isotropic and aniostropic materials, see text). 
Both of the two complex-conjugate values $\nu$ and $\nu^{*}$ are acceptable because each corresponds to a different time dependence ( $e^{j \omega t}$ and $e^{-j \omega t}$, respectively).

Figure 10 shows a useful nomogram that can be used to determine the optical properties of a medium using the TRM at normal and $45^{\circ}$ angle of incidence. This nomogram is obtained by the superposition of the $z$ and $w$ planes in Fig. 7 . Similar nomograms can be constructed that apply when reflectance measurements are made at normal and any general angle of incidence. The technique can also be generalized to measurements of reflectance at any two angles of incidence. ${ }^{13-16}$

The foregoing TRM can be extended to apply to anisotropic media. For a uniaxial crystal, the reflectances of $s$-polarized light at normal and oblique incidence can be measured on a crystal face cut parallel to the optic axis. When the optic axis is in the plane of incidence, the reflection is governed by the ordinary complex refractive index $N_{o}$ only, which can be determined exactly as described before for the case of an isotropic medium. If the measurements are repeated with the optic axis perpendicular to the plane of incidence (i.e., oriented in the $s$ direction), the complex extraordinary refractive index $N_{e}$ can likewise be determined. For a biaxial absorbing crystal, with three principal axes $x, y$, and $z$, all of the optical properties can be determined by repeated application of the TRM on two crystal faces each containing a different pair of principal axes, e.g., $x y$ and $y z$. If three sets of reflectance measurements at normal and oblique incidence are made, with the crystal oriented such that each principal axis is brought in turn in alignment with the linear vibration of incident $s$ polarized light, all of the three complex principal refractive indices $N_{x}, N_{y}$, and $N_{z}$ can be obtained, using exactly the same procedure appropriate to the case of an isotropic medium.

${ }^{1}$ A. Fresnel, "Mémoire sur la loi des modifications que la réflection imprime a la lumière polarisêe," in Oeuvres Complètes de Fresnel, Vol. 1, H. Senarmont, E. Verdet, and L. Fresnel, 1866, pp. 767-775 (Johnson Reprint Corporation, New York, 1965).

${ }^{2}$ See, for example, R. M. A. Azzam and N. M. Bashara, Ellipsometry and Polarized Light (North-Holland, Amsterdam, 1977), Sec. 4.2 .

'It will be significant to study the relationship between each Fresnel coefficient and the refractive index ratio $\nu$ as a conformal mapping between two complex planes.

${ }^{4}$ This choice of the $p$ and $s$ directions follows the Nebraska (Muller) conventions. See, R.H. Muller, "Definitions and conventions in ellipsometry," Surf. Sci. 16, 14-33 (1969).

"It is important to observe that any pair of Fresnel coefficients can be directly interrelated by the elimination of their common variable

'In the Nebraska (Muller) conventions, the $e^{j \omega t}$ time dependence is chosen (see Ref. 4).

${ }^{7}$ Zero reflection at normal incidence $(z=0)$ leads, as expected, to zero reflection at oblique incidence $(w=0)$. Notice that $z \rightarrow 0$ as $\nu \rightarrow$ 1 , i.e., when the difference between the refractive indices of the two media that surround the interface tends to zero.

8Points along the real axis of the $z$ plane represent normal-incidence reflection of $s$-polarized light at a dielectric/dielectric interface $(\nu$ real, hence $z$ real) and the image points in the $w$ plane represent the reflection of that light from the same interface at an angle $\phi$. The oblique reflection is total (i.e., $|w|=1$ ) if the refractive index ratio $\nu \geqslant \sin \phi$ or $z \geqslant(1-\sin \phi) /(1+\sin \phi)$

${ }^{9}$ Total reflection at normal incidence $(|z|=1)$ leads, as expected, to total reflection at oblique incidence $(|w|=1)$. Notice that $|z| \rightarrow$ 1 as $\nu \rightarrow \infty$ which happens when light is reflected from the interface between a medium with a finite refractive index and another which is perfectly conducting.

${ }^{10}$ These lines are the loci of constant normal-incidence phase shift.

${ }^{11}$ These circles are the loci of constant normal-incidence (amplitude or intensity) reflectance.

${ }^{12} \mathrm{O}$. S. Heavens, Optical Properties of Thin Solid Films.(Dover, New York, 1965), p. 85.

${ }^{13}$ Various two reflectance methods for determining the optical properties of materials have been previously described. See, for example, Refs. 14-16. Although we assume $s$-polarized light, identical procedures apply for $p$-polarized light. The sensitivity of the TRM is known to be poorer with the $s$ polarization than with the $p$ polarization, but straightforward extension of the method to anisotropic media is possible only with $s$-polarized light (as is illustrated in this section).

${ }^{14}$ I. Simon, "Spectroscopy in the infrared by reflection and its use for highly absorbing substances," J. Opt. Soc. Am. 41, 336-345 (1951).

${ }^{15}$ S. P. F. Humphreys-Owen, "Comparison of reflection methods for measuring optical constants without polarimetric analysis, and proposal for new methods based on the Brewster angle," Proc. R. Soc. Lond. 77, 949-957 (1961).

${ }^{16} \mathrm{~W}$. R. Hunter, "Errors in using the reflectance vs angle of incidence method for measuring optical constants," J. Opt. Soc. Am. 55, 1197-1204 (1965). 